\title{
LXXVI. The absorption of homogeneous $\beta$ rays
}

\section{R.W. Varder M.A.}

To cite this article: R.W. Varder M.A. (1915) LXXVI. The absorption of homogeneous $\beta$ rays , Philosophical Magazine Series 6, 29:173, 725-733, DOI: 10.1080/14786440508635354

To link to this article: http://dx.doi.org/10.1080/14786440508635354

曲 Published online: 08 Apr 2009.

Submit your article to this journal $₫$

Џ Article views: 3

Q View related articles $₫$

4 Citing articles: 31 View citing articles $\asymp$ 


\section{$\left[\begin{array}{ll}725 & ]\end{array}\right.$}

LXXVI. The Alsorption of Homogeneous $\beta$ Rays.

$B y$ R. W. VArder, M.A. (Cape) *

$r$ WHE experiments described in this paper were made to investigate as accurately as possible the form of the ionization absorption curve when very homogeneous $\beta$ rays pass through a standard substance like aluminium, and also to test whether there is any simple relation connecting the absorption with either the velocity or energy of the $\beta$ particle.

The apparatus used is shown in fig. 1.

Fig. 1.

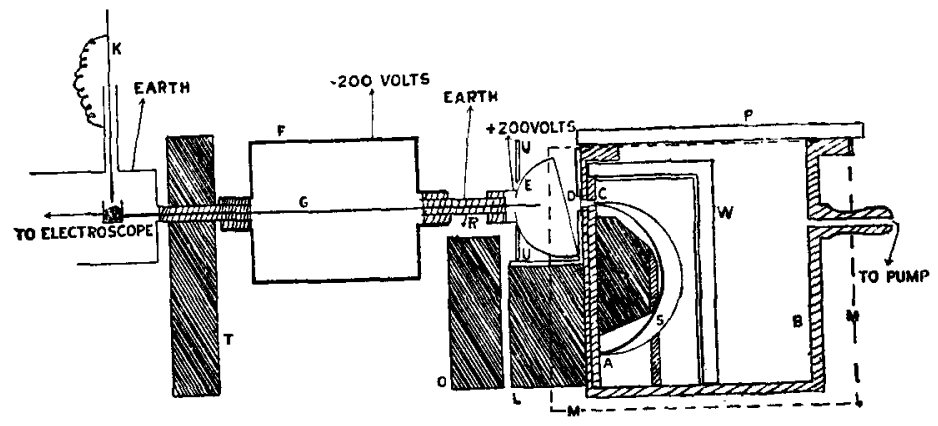

B is a brass box about $3 \mathrm{~cm}$. wide and $10 \mathrm{~cm}$. high. It was placed between the poles $(17 \times 10 \mathrm{~cm}$.) of a large electromagnet. The position of the pole-pieces is denoted by the broken line MM. Rays from a thin-walled tube A filled with radium emanation describe circles in the magnetic field; a pencil of homogeneous $\beta$ rays passing through the slit $\mathrm{S}$ could be concentrated at $\mathrm{C}$. The possibility of obtaining very homogeneous $\beta$ rays by this method has been shown by Rutherford and Robinson $\dagger$, who employed it to determine the $\beta$ ray spectrum of radium $B$ and $C$. In the present experiments the relative positions of the source $A$, the slit $S$, and the opening $\mathrm{C}$ were arranged to obtain a concentrated and nearly homogeneous beam at $\mathrm{C}$. The rays there passed through a rectangular slit $1 \mathrm{~cm} . \times 2 \mathrm{~mm}$. in a brass plate and through the mica window D. A ground-glass plate $P$ placed on the top of the box made it airtight. In order to prevent scattering and diminution in intensity, the airpressure in B was reduced to about $1 \mathrm{~cm}$. of mercury. The window $\mathrm{D}$ had a stopping power corresponding to about $2 \mathrm{~cm}$. of air at N.T.P. In this way a strong pencil of $\beta$ rays

* Communicated by Sir E. Rutherford, F.R.S.

† Rutherford and Robinson, Phil. Mag. Oct. 1913. 
was obtained for which the average value of $\mathrm{H} \rho$, where $\mathrm{H}$ is the magnetic field and $\rho$ the radius of curvature, could be accurately determined. The value of $\rho$ was fixed at $3 \cdot 19 \mathrm{~cm}$. in all the experiments, and readings could be obtained up to about $\mathrm{H} \rho=12,000$ gauss $\mathrm{cm}$. From the dimensions of the source, slit, and opening, it was calculated that the variation of $\mathrm{H} \rho$ for the issuing rays was less than 3 per cent.

The lead blocks L helped to screen the ionization apparatus from the effects of the $\gamma$ rays. $E$ is a hemispherical chamber of $2 \mathrm{~cm}$. radius made of coarse copper gauze covered with copper leaf and thin tissue-paper. This vessel was charged to +200 volt. $\mathrm{F}$ is a brass cylindrical vessel charged to -200 volt. A copper electrode $G$ passed through two ebonite plugs at the ends of $F$ and terminated at one end in $E$. The other end of $G$ was connected to a Wilson-Kaye electroscope, which was screened by the lead block T. $\mathbf{K}$ is a key for earthing $G$, and $R$ a guard-ring. A wooden frame $W$, coated on the inside with celluloid, minimised the effect of reflected $\beta$ radiation. The vessel $F$ was put in to balance partly the $\gamma$ ray ionization in $\mathbf{E}$. By altering the position of the lead block $O$ relative to the vessel $F$, the $\gamma$ ray ionization in $F$ could be varied so as to neutralize nearly the $\gamma$ ray ionization in $\mathrm{E}$. Pieces of celluloid $\mathrm{U}$ were placed in front, below, and at the back of $E$ as shown. This was done to minimise the reflexion effects of the $\beta$ rays.

The magnetic fields were measured by comparing the throws of a ballistic galvanometer when an exploring coil was removed from the field, with the throw prodnced when a known current was broken in the primary of a standard mutual induction. The current in the primary of the mutual induction was measured on a standardized ammeter, and the coils used had also been standardized in some previous work. In this way the ficlds were measured to about one part in 400. By means of a fluxmeter it was found that the fields were practically uniform over the region traversed by the rays in the box. A given field could always be reproduced by setting the current in the magnetcoils to a certain value and reversing it a number of times.

In taking a reading, the current through the magnet-coils was set at a particular value and reversed a number of times to reach a cyclic state. The vessel $B$ was exhausted by means of a Flenss pump. The block $O$ was adjusted so that the motion of the electroscope-leaf was slow when a thick aluminium plate was placed over the flat face of $\mathrm{E}$. Readings were taken of the rate of motion of the electroscope-leaf 
when various thicknesses of foil were placed over E. The current was then reversed so that no $\beta$ rays passed through $\mathrm{C}$, and similar readings were taken. $\mathrm{A}$ curve of the type AA (fig. 2) was obtained when the field was direct, and one of type $\mathrm{BB}$ when the field was reversed; the two curves

Fig. 2.

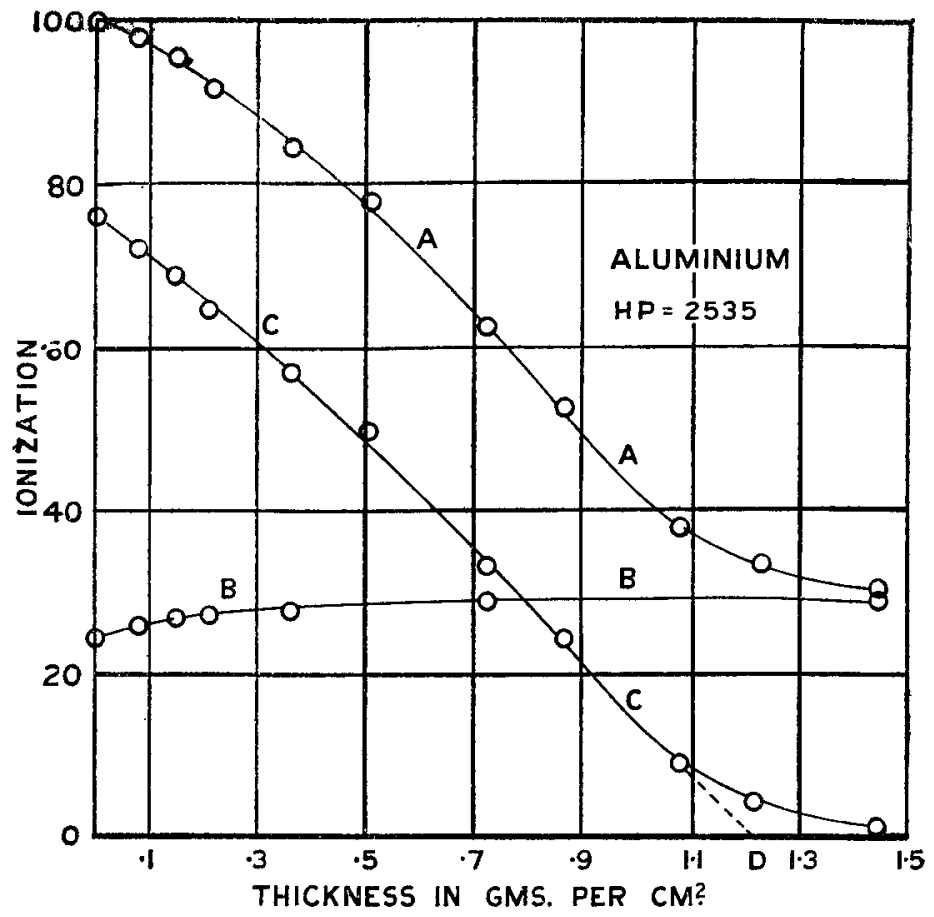

coincided for a very thick absorbing screen. The curve AA represents the combined $\beta$ and $\gamma$ ray effect in $\mathrm{E}$, the curve $\mathrm{BB}$ represents the $\gamma$-ray effect alone, so that the curve $\mathrm{CC}$, which is obtained by subtracting curve $B B$ from the curve $A A$, represents the curve of $\beta$ ray absorption. The interpretation of the curve $\mathrm{BB}$ will be discussed later.

After a small thickness of matter had been traversed, an approximately linear relation (see curve $\mathrm{CC}$ ) was found to exist between the ionization and the thickness of the absorbing screen. The curves CC for various velocities are shown in figs. 3 and 4. 
The initial drop of the curves varied with the velocity; this is very probably due to the partial absorption of some of the rays at the edges of the opening $C$ (fig. 1). After this initial drop, the curves are similar in type. In the diagrams the thickness of aluminium is expressed in grams per sq. $\mathrm{cm}$. This thickness in the diagrams is uncorrected for the thickness of the mica window $\mathrm{D}$. The initial rise of the curve $\mathrm{BB}$ (fig.2) is due to $\beta$ radiation excited in the screens under the conditions of the experiment by the $\gamma$ rays from A. The current

Fig. 3.

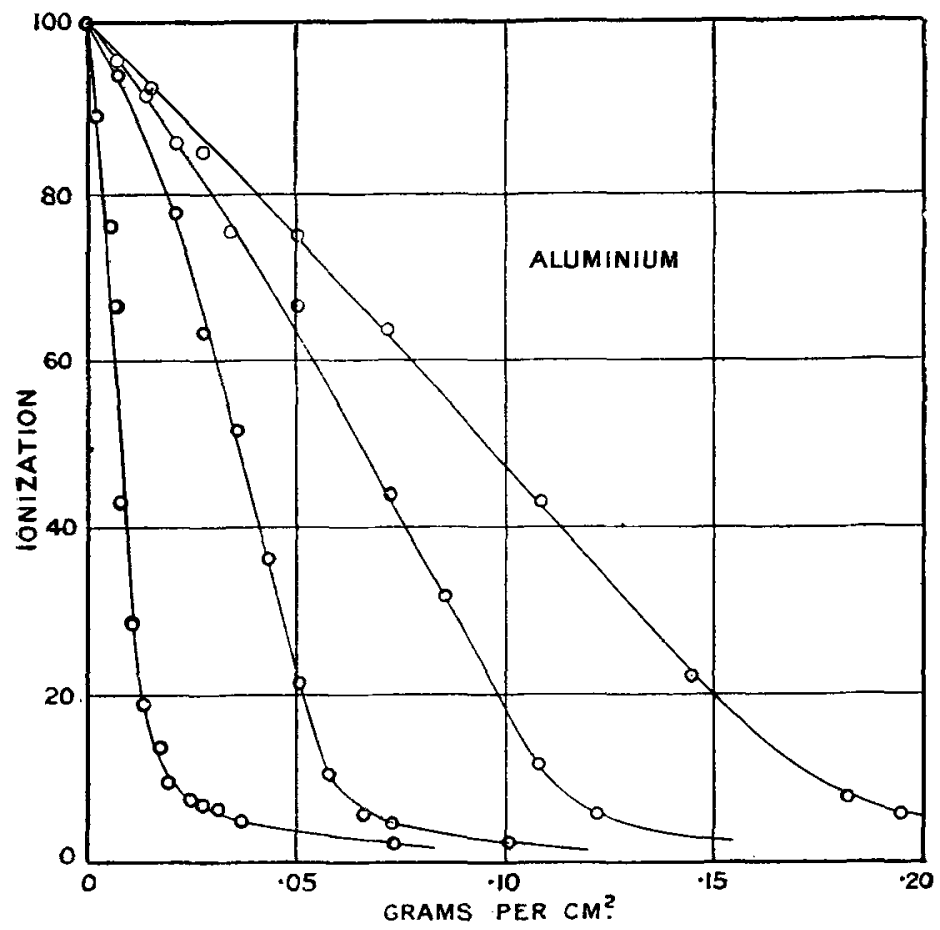

increased with the thickness of the absorbing sereens fairly rapidly at first and then very slowly. This effect is very important when the ratio of the $\beta$-ray ionization in $\mathrm{E}$ to the $\gamma$-ray ionization is small. In the experiment with $\mathrm{H} \rho=2000$ gauss $\mathrm{cm}$.- the strong part of the spectrum-this ratio was about 2. In this way absorption curves were taken from 
$\mathrm{H} \rho=1380$ to $\mathrm{H} \rho=11500$ gauss $\mathrm{cm}$. Curves for slower rays are shown in fig. 3, and for faster rays in fig. 4 . $\quad \beta$ particles could, however, be detected with certainty up to $\mathrm{H} \rho=16000$, when they were so swift as to be able to pass through more than $1 \mathrm{~cm}$. of aluninium. Readings at this point were inconvenient on account of the high currents in the magnetcoils necessary to produce the required field.

Fig. 4.

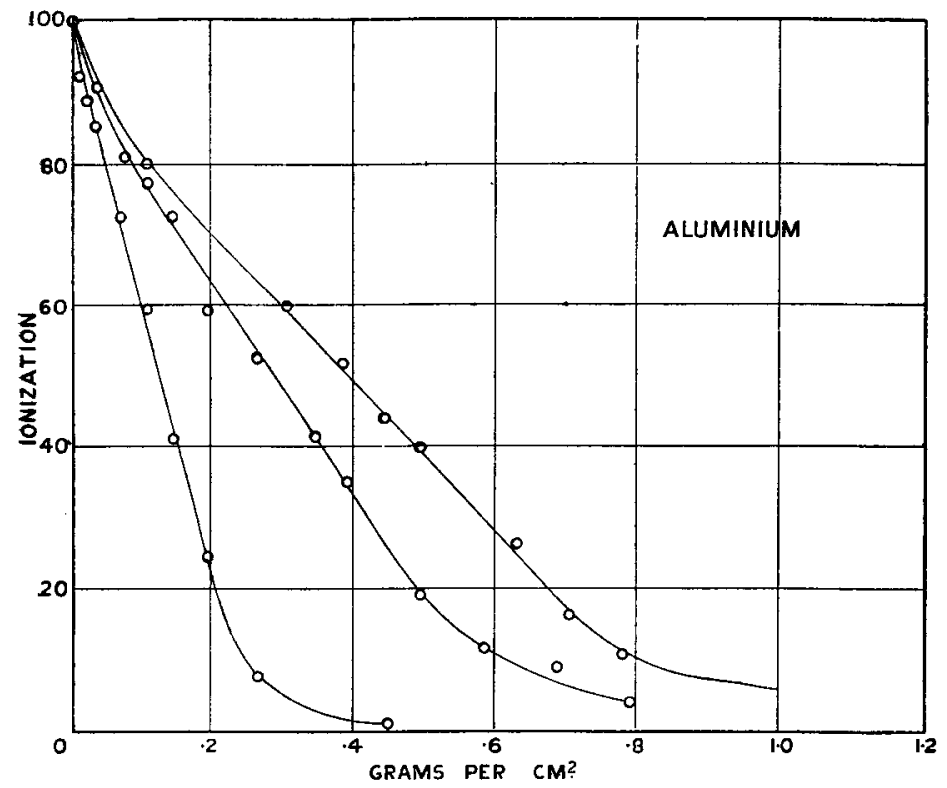

By producing the linear part of curve $\mathrm{CC}$ (fig. 2) to cut the axis of $\mathrm{D}$, we get the thickness of matter, $O D$, which would be traversed by the $\beta$ particles if the law of absorption had continued as in the linear part of the curve. The distance OD might, on analogy of the Bragg $\alpha$-ray curves, be called the "range" of the $\beta$ particle in aluminium. It gives the thickness of aluminium in which most of the $\beta$ particles are stopped. Such a quantity may prove useful in some experiments in deducing the velocity of $\beta$ rays from absorption curves. A curve showing the range of $\beta$ rays in aluminium at various velocities is given in fig. 5 . 
In discussing this question with Dr. Bohr, he informed me that he had deduced from theoretical considerations that the

Fig. อ.



loss of onergy $(d \mathrm{~T})$ of a $\beta$ particle in going through a thickness of matter $(d x)$ should be given by

$$
\frac{d^{\prime} \mathrm{T}}{d x}=-\frac{\mathrm{K}}{\beta^{2}}
$$

where

$$
\mathrm{T}=m_{0} c^{2}\left(\left(1-\beta^{2}\right)^{-\frac{1}{2}}-1\right)
$$

is the kinetic energy of the particle.

$\beta=$ ratio of velocity of $\beta$ particle to the velocity of light.

$K$ is a function of the velocity which increases slowly with increasing velocity. If we assume $K$ is constant, and 
integrate to find the thickness of matter $R$ in which the kinetic energy is destroyed, we get

$$
\begin{aligned}
\int_{0}^{\mathrm{R}} d x & =\int_{0}^{\beta}-\frac{1}{\mathrm{~K}} \beta^{2} d \mathrm{~T} \\
& =\int_{0}^{\beta}-\frac{c^{2} m_{0}}{\mathrm{~K}} \beta^{2} d\left\{\left(1-\beta^{2}\right)^{\left.-\frac{1}{2}\right\}},\right. \\
\mathrm{R} & =\frac{c^{2} m_{0}}{\mathrm{~K}}\left[\left(1-\beta^{2}\right)^{\frac{1}{2}}+\left(1-\beta^{2}\right)^{-\frac{1}{2}}-2\right] \\
& =\frac{c^{2} m_{0}}{\mathrm{~K}} \mathrm{~A},
\end{aligned}
$$

where. $\quad A=\left[\left(1-\beta^{2}\right)^{\frac{1}{2}}+\left(1-\beta^{2}\right)^{-\frac{1}{2}}-2\right]$.

In the following table in column $I$. is given the value of $\mathrm{H} \rho$ in gauss cm. In column II. the value of $\beta$ deduced from the relativity formula

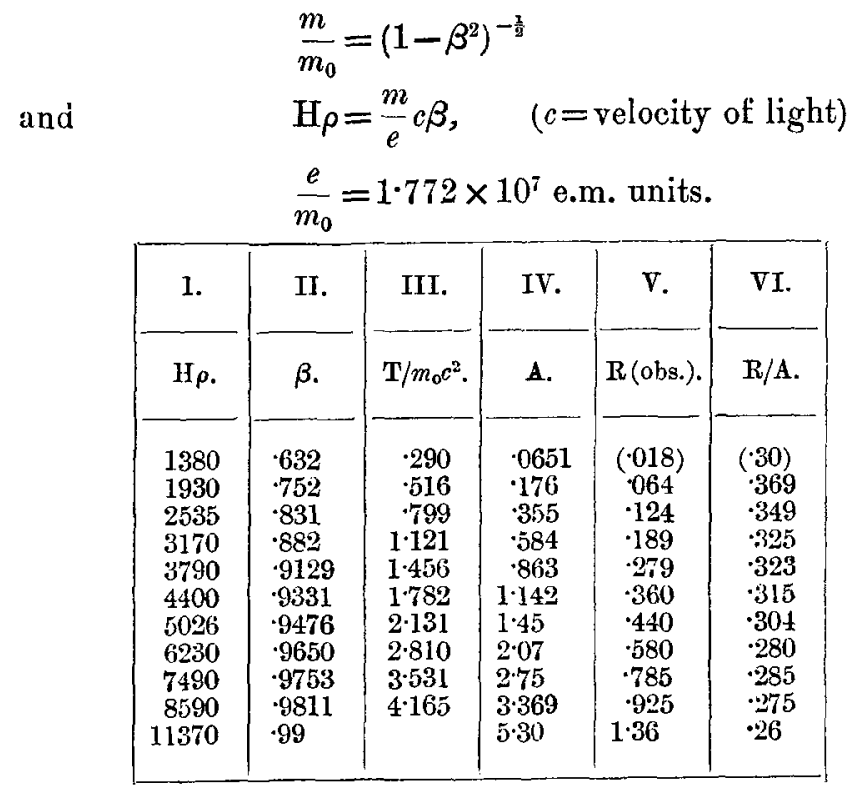

The values of $\mathrm{R}$ (obs.) are expressed in gm. per sq. $\mathrm{cm}$.

In column III. the value of $T / m_{0} c^{2}$ is given. In column IV. the value of $A$. In column $V$. the value of $R$, the range observed from experiments, and in column VI., R(obs.)/A. 
$\frac{\mathbf{R} \text { (obs.) }}{\mathrm{A}}$ ought to be equal to $c^{2} m_{0} / \mathrm{K}$. With the exception of the first value, we see that $\frac{R \text { (obs.) }}{A}$ decreases slowly with increasing velocity, as should be expected from theory since $\mathrm{K}$ increases slowly with increasing velocity.

The fact that the aluminium absorption curve is approximately linear must result from a chance balancing of the opposing effects of scattering and diminution of velocity. If we had no scattering or straggling we should expect to have a curve similar in shape to a Bragg a-ray curve.

For a substance like paper, which contains only elements of low atomic weight, the effect of scattering is less important than for aluminium, so that a greater fraction of $\beta$ rays will penetrate a given thickness of matter. This is seen from the fact that the absorption curve for filter-paper (fig. 6) is

Fig. 6.

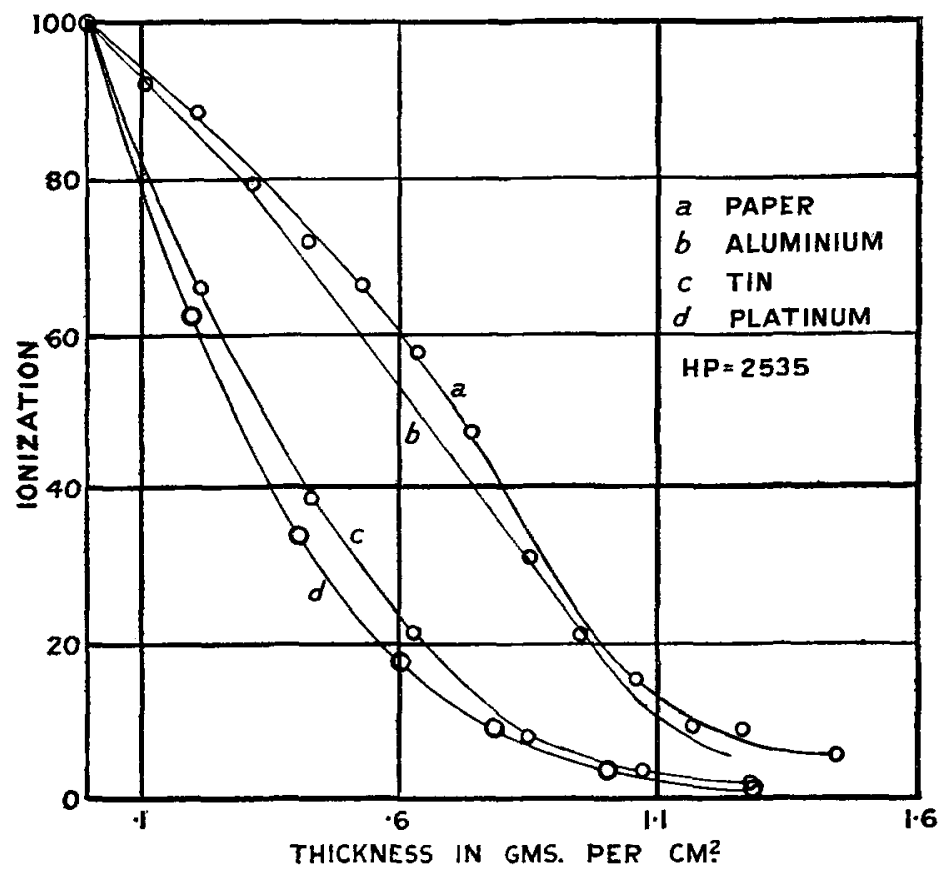

concave to the origin. For substances, like tin and platinum, of high atomic weight fewer $\beta$ particles penetrate a given thickness, and the curves are convex towards the origin (fig. 6). 
W. Wilson* has discussed the question of absorption of swift $\beta$ rays by aluminium in two papers. The results given in his first communication agree approximately with those given in the present paper. He examined this question again in a second investigation, in which he believed that he obtained more homogeneous $\beta$ rays. In this paper he finds that the absorption curve for fast rays in aluminium rises to a maximum for a small thickness of matter and then decreases in an approximately linear relation. His method of measuring the ionization due to the $\beta$ rays was to subtract the reading of the electroscope with a thick screen from the reading with the screen in which the absorption of the $\beta$ rays was under examination. Thus he assumes that the $\gamma$ radiation produces the same quantity of secondary $\beta$ radiation in a thick plate as in a thin plate. In general this is not the case. The conditions of his experiment are not sufficiently clear to form an idea of the magnitude of this correction ; for it will depend on what material he placed in front of his ionization vessel (behind the aluminium screen). In my experiments, with celluloid in this position, the secondary $\beta$-ray effect was greater with a thick aluminium plate than with a thin one, as is shown in curve BB (fig. 2). If this were the case in Wilson's experiment, the rise of his absorption curve to a maximum receives a simple explanation. This is rendered the more probable since he mentions that the $\gamma$-ray effect was relatively strong, thus giving a relatively great importance to the secondary $\beta$ rays from the absorbing screens. I have tried to obtain his results by narrowing the slits to produce greater purity of rays, but have been unsuccessful. In Wilson's experiment the air was not exhausted from the apparatus. To test the effect of this, readings were taken with air in the box B (fig. 1), but the only obvious effect was a diminution in intensity of the $\beta$ radiation.

In conclusion I wish to thank Prof. Sir Ernest Rutherford for suggesting this research and for many valuable suggestions during its progress. I also wish to thank Dr. N. Bohr for his great interest and help in connexion with the theory of absorption of $\beta$ particles.

The Physics Laboratory,

Victoria University of Manchester, March 4, 1915.

* W. Wilson, Proc. Roy. Soc. A. lxxxii. p. 612 (1909); lxxxvii. p. 310 (1912). 